\title{
Lattice QCD results on chemical freeze-out
}

\author{
Claudia Ratti ${ }^{1} \star \star$ \\ ${ }^{1}$ Department of Physics, University of Houston, Houston, TX 77204, USA
}

\begin{abstract}
The latest lattice QCD results on fluctuations of conserved charges at finite temperature and density are reviewed, and connected to the experimental moments of their net-multiplicity distributions, in order to describe the chemical freeze-out from first principles.
\end{abstract}

\section{Introduction}

The chemical freeze-out is the moment, in the evolution of a heavy-ion collision, at which all inelastic collisions between particles cease. The chemical composition is fixed at this point, which means that both measured particle multiplicities and fluctuations carry information about this particular moment in the evolution of the system. In particular we are interested in determining the freeze-out temperature and chemical potential, and establishing how far the freeze-out line is from the transition line of QCD.

The first thing to look at, to characterize the chemical freeze-out, is therefore particle yields. The Hadron Resonance Gas (HRG) model has been very successful in fitting particle yields and ratios over nine orders of magnitude [1-5]. However, the spectrum used as an input in the HRG model is still being regularly updated, and many missing states are expected to be relevant to the thermodynamics around the transition temperature [6-9]. This introduces an uncertainty in the freeze-out parameters, since the decays modes of these additional states are not known. For this reason, a model-independent approach would be desirable.

All of this motivates the idea of finding a way to extract the freeze-out parameters from first principles, namely by comparing experimental results to lattice QCD directly. Lattice simulations are the most established tool to address QCD in its non-perturbative regime. Lattice QCD is a numerical method, and as such affected by statistical and systematic uncertainties. Due to a steady and continuous improvement in computer resources, numerical algorithms and our physical understanding which manifests itself in physical techniques (e.g. the Wilson-flow scale setting introduced in Ref. [10]), the lattice results which are being produced today reach an unprecedented level of accuracy. This allows a quantitative comparison to experimental observables for the first time in heavy ion physics.

\section{Thermal fits to particle yields and ratios}

The first thing to look at, to characterize the chemical freeze-out, is therefore particle yields and ratios. The particle yields are given by this simple formula, plus a contribution coming from resonance

\footnotetext{
^e-mail: cratti@uh.edu
} 
decays, in the HRG model:

$$
N_{i}=-\frac{\partial \ln Z_{i}}{\partial \mu}=\frac{g_{i} V}{2 \pi^{2}} \int_{0}^{\infty} \frac{p^{2} d p}{\exp \left[\left(E_{i}-\mu_{i}\right)\right] / T \pm 1}
$$

where $E_{i}=\sqrt{p^{2}+m_{i}^{2}}, \mu_{i}=\mu_{B} B_{i}+\mu_{S} S_{i}+\mu_{Q} Q_{i}$, and $g_{i}$ is the degeneracy of particle $i$. This kind of fits has been performed for a long time and has been very successful in describing the multiplicity of particles produced in heavy-ion collisions. Changing the collision energy, it is possible to draw the freeze-out line in the $\left(T, \mu_{B}\right)$-plane. An example of such a fit for the LHC energies, together with the chemical freeze-out line, is shown in Fig. 1. Recently, the possibility of a flavor hierarchy in
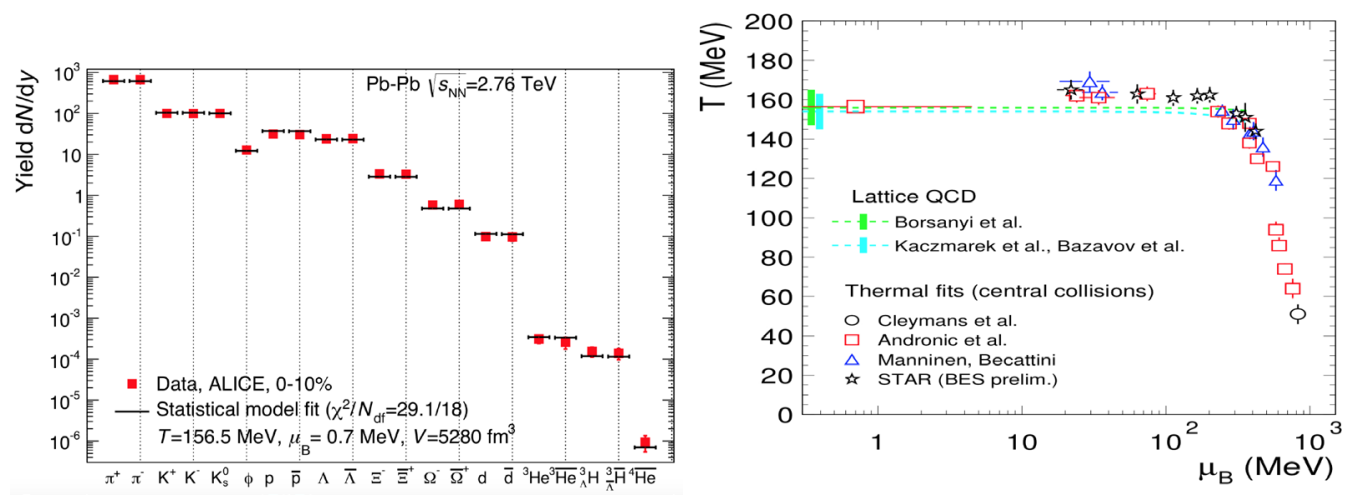

Figure 1. Left: Best fit of hadron multiplicities in central $(0-10 \%) \mathrm{Pb}-\mathrm{Pb}$ collisions at the LHC ALICE experiment [11]. Right: The phase diagram of strongly interacting matter with the points representing the thermal fits of hadron yields at various collision energies [1-5]. The crossover $T$ values from lattice QCD [12,13] are shown as bands at small $\mu_{B}$ values. The dashed lines represent lattice QCD calculations of the curvature parameter $[14,15]$.

the freeze-out temperatures has been discussed $[16,17]$, with the freeze-out temperature for strange hadrons being larger than the one for light hadrons. Among the explanations of this phenomenon, I will focus here on the one proposed in Ref. [8]. A disagreement was found between the ratio of strange to baryon chemical potential obtained on the lattice and the one predicted by the HRG model based on the resonance spectrum listed in the particle data book. The agreement can be reached when additional states are added to the spectrum, which have not yet been detected but are predicted by the quark model. Since the strange chemical potential enters the thermal fits, this will affect the freeze-out parameters, potentially reducing the strangeness freeze-out temperature and bringing it closer to the light quark one. In Ref. [9] we performed a systematic study of the contribution of different hadronic species to the QCD pressure, separated according to their flavor and baryon number content. They are shown in Fig. 2. This has allowed us to determine which hadronic families are still missing resonances, and indeed most groups of hadron need more states than the ones listed as "known" in the PDG. This will impact the thermal fits, since the decay modes of these states are not known. 

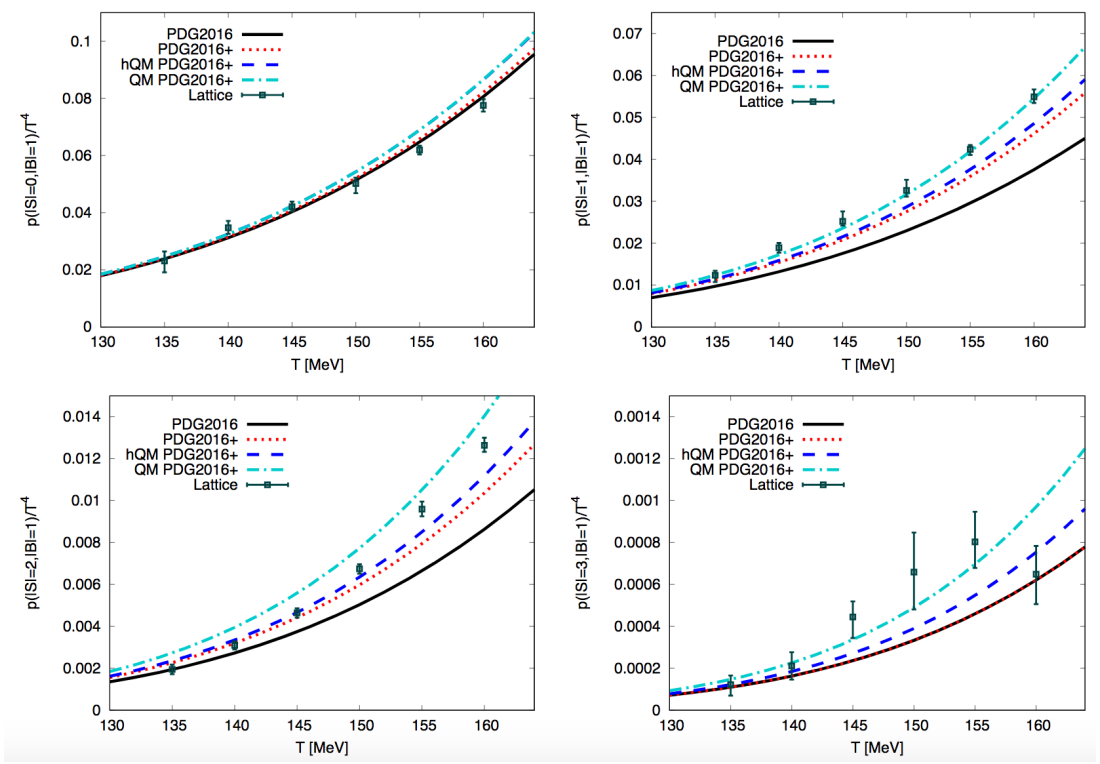

Figure 2. Comparison between the lattice results for the partial pressures and the HRG model predictions. Upper panels: non-strange baryons (left), $|\mathrm{S}|=1$ baryons (right). Lower panels: $|\mathrm{S}|=2$ baryons (left), $|\mathrm{S}|=3$ baryons (right). The points are the lattice results, while the curves are PDG2016 (solid black), PDG2016+ (including one star states, red dotted), PDG2016+ and additional states from the hQM (blue, dashed) [18, 19], PDG2016+ and additional states from the QM (cyan, dash-dotted) [20, 21].

\section{Fluctuations of conserved charges}

Fluctuations of conserved charges are defined as

$$
\chi_{\text {lmn }}^{B S Q}=\frac{\partial^{l+m+n}\left(p / T^{4}\right)}{\left(\partial \mu_{B} / T\right)^{l}\left(\partial \mu_{S} / T\right)^{m}\left(\partial \mu_{Q} / T\right)^{n}} .
$$

They can be calculated on the lattice as combinations of quark flavor fluctuations, through the following relationship between chemical potentials:

$$
\begin{aligned}
\mu_{u} & =\frac{1}{3} \mu_{B}+\frac{2}{3} \mu_{Q} \\
\mu_{d} & =\frac{1}{3} \mu_{B}-\frac{1}{3} \mu_{Q} \\
\mu_{s} & =\frac{1}{3} \mu_{B}-\frac{1}{3} \mu_{Q}-\mu_{S} .
\end{aligned}
$$

The relevance of fluctuations for the physics of heavy ion collisions has been increasing in recent years. The first continuum-extrapolated second order fluctuations of conserved charges, evaluated at physical values for the quark masses, were presented in Ref. [22] (almost-physical quark mass results are shown in Ref. [23], heavier quark mass results are shown in Ref. [24]) and later extended to selected fourth-order fluctuations and correlations [25, 26].

The most interesting feature of fluctuations is that they can be related to the moments of the distribution of the corresponding conserved charges (mean $M$, variance $\sigma^{2}$, skewness $S$ and kurtosis 
$\kappa)$ through the following formulas

$$
\begin{aligned}
M=\chi_{1} & \sigma^{2}=\chi_{2} \\
S=\chi_{3} / \chi_{2}^{3 / 2} & \kappa=\chi_{4} / \chi_{2}^{2} .
\end{aligned}
$$

Usually ratios are defined, so that the volume factor cancels out in the theoretical definition of fluctuations, and they become functions only of $T$ and $\mu_{B}$ :

$$
\begin{aligned}
M / \sigma^{2} & =\chi_{1} / \chi_{2} & & S \sigma=\chi_{3} / \chi_{2} \\
S \sigma^{3} / M & =\chi_{3} / \chi_{1} & & \kappa \sigma^{2}=\chi_{4} / \chi_{2} .
\end{aligned}
$$

If the detector could cover the entire solid angle, none of the conserved charges would fluctuate. But if we look at a sub-system, limited by the acceptance of the detector, the conserved charges can fluctuate in and out of this sub-system, which then can be treated as a Grand Canonical Ensemble. Therefore, the detector should have a rapidity window small enough that charge conservation does not suppress the signal, but large enough that the system can be treated as a thermodynamic ensemble.

The rapidity dependence is only one of the effects that we need to study in order to have a meaningful comparison between lattice QCD and experiments. Other possible experimental sources of non-thermal fluctuations are corrected for in the STAR data analysis: the centrality-bin-width correction method minimizes effects due to volume variation because of finite centrality bin width (such effects have been studied e.g. in [27-29]); the moments are corrected for the finite reconstruction efficiency based on binomial probability distribution [30]. Interactions in the hadronic phase and non-equilibrium effects might affect fluctuations [32-35]; the consistency between the freeze-out parameters obtained from the analysis of fluctuations of different quantum numbers, such as electric charge and baryon number, represent a fundamental test of the equilibrium scenario.

One should also notice that, experimentally, only the net-proton multiplicity distribution is measured, as opposed to the lattice net-baryon number fluctuations. However, taking into account the effects of resonance feed-down and isospin randomization [36, 37], one can show that the net-proton and net-baryon number fluctuations are numerically very similar, at least in the case of low-order fluctuations [38].

The first comparisons between lattice and experiment were performed by the WB collaboration in $2013[39,40]$ and used $\chi_{3} / \chi_{1}$ for baryon number to extract an upper limit for the freeze-out temperature and $\chi_{2} / \chi_{1}$ from the same conserved charge to get the baryonic chemical potential for the top 4 collision energies at RHIC [41, 42]. From this comparison, a maximum freeze-out temperature from fluctuations of $151 \pm 4 \mathrm{MeV}$ was found. The chemical potentials from $\chi_{2} / \chi_{1}$ for baryon number were also obtained. An independent analysis based on a comparison between lattice QCD results and experimental data for the electric charge $\chi_{2} / \chi_{1}$ found perfect consistency between the freeze-out chemical potentials. These results also agree with an independent study of the same fluctuations in the framework of the Hadron Resonance Gas model [43]. A freeze-out temperature of $147 \pm 2 \mathrm{MeV}$ at the highest RHIC collision energy was obtained in Ref. [44] from a study of the ratio $\chi_{1} / \chi_{2}$ for electric charge divided by the baryon number one. This analysis also provided an upper limit on the curvature for the freeze-out line, which turns out to be very small.

More recently, preliminary results have been shown for a combined fit of $\chi_{1} / \chi_{2}$ for electric charge and baryon number, which allows to extract both freeze-out $T$ and $\mu_{B}$ at a given collision energy [45]. The results are shown in the left panel of figure 3 . The colored lines are the trajectories in the $\left(T, \mu_{B}\right)$ plane which satisfy the experimental value for $\chi_{1} / \chi_{2}$ for the two conserved charges. The points where they cross yield the freeze-out $T$ and $\mu_{B}$ from fluctuations. Notice that the black points in the plot are obtained from the independent analysis of the same data done in the HRG model in Ref. [43], which perfectly agrees with the lattice results. On the same plot, the current understanding of the 
QCD phase diagram from lattice simulations is shown [15], together with the isentropic trajectories that were reconstructed starting from the freeze-out points [46].
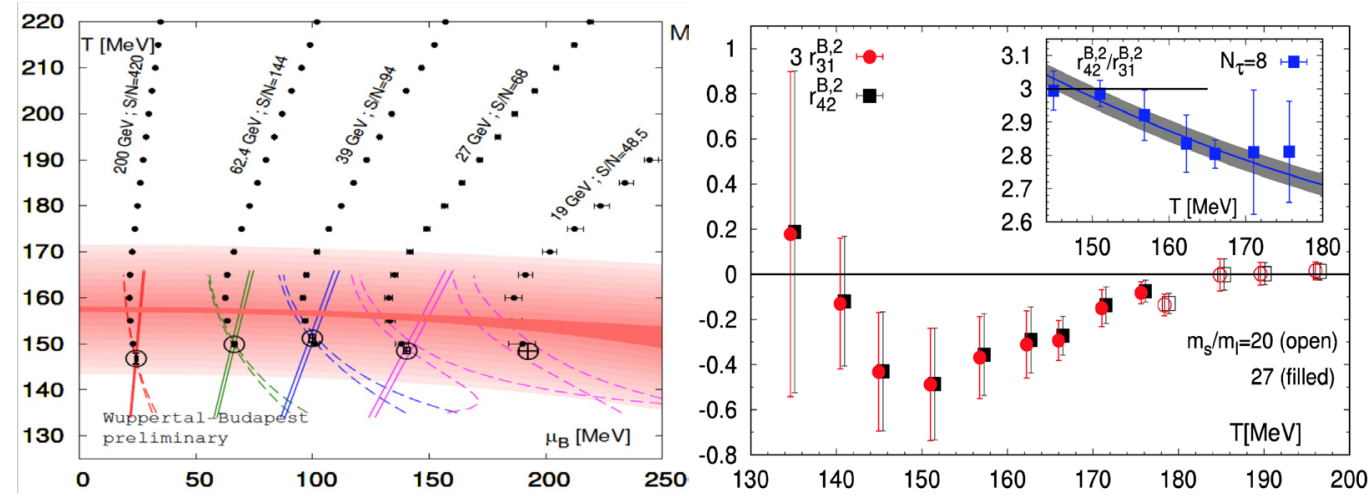

Figure 3. Left: Preliminary results of the WB collaboration [45]. The colored full and dashed lines are the contours at constant mean/variance ratios of the net electric charge from lattice simulations. The contours that correspond to STAR data intersect in the freeze-out points of Ref. [43]. The red band is the QCD phase diagram shown in Ref. [15]. Also shown are the isentropic contours that match the chemical freeze-out data [46]. Right: Taylor expansion coefficients for $\chi_{3} / \chi_{1}$ multiplied by 3 (red), and $\chi_{4} / \chi_{2}$ (black) as functions of the temperature from Ref. [49].

Preliminary results for net-proton fluctuations up to second order at the LHC have been presented recently [47]. The authors of Ref. [48] have performed an analysis, assuming that the lower moments follow the Skellam distribution. In that case, it is possible to express the second-order moments in terms of particle yields, for which experimental data exist for a long time. The analysis was focused on the ratios $\chi_{2}^{B} / \chi_{2}^{S}, \chi_{2}^{B} / \chi_{11}^{Q S}$ and $\chi_{11}^{B S} / \chi_{2}^{S}$. The obtained freeze-out temperature was slightly larger than then one obtained from the fluctuation analysis at RHIC.

In general, the higher order fluctuations are more affected by some of the non-thermal sources of fluctuations discussed above. Nevertheless, they can be calculated on the lattice at finite density by means of a Taylor expansion and compared to the experimental results, to test whether QCD can explain some of the trends seen in the data. For example, the authors of Ref. [49] have expanded the baryonic $\chi_{3} / \chi_{1}$ and $\chi_{4} / \chi_{2}$ in Taylor series in powers of $\mu_{B} / T$. The Taylor expansion coefficients for the two are plotted in the right panel of Fig. 3: the red points are the coefficient for $\chi_{3} / \chi_{1}$ multiplied by 3 , while the black points are the coefficient for $\chi_{4} / \chi_{2}$. It is evident that $\chi_{4} / \chi_{2}$ falls three times faster than $\chi_{3} / \chi_{1}$ with increasing chemical potential. The experimental data for these quantities show the same trend.

The next question is whether the third conserved charge, namely strangeness, shows the same consistency which has been found between electric charge and baryon number with respect to the chemical freeze-out parameters. Full strangeness fluctuations are difficult to measure, due to the multistrange particles. The STAR collaboration recently finalized the results for the kaon fluctuations [50]. Kaon fluctuations are very sensitive to the freeze-out parameters [51] and could represent a first step to extract the strangeness freeze-out temperature. Recently, a method to isolate the kaon fluctuations on the lattice, based on the HRG model, was proposed [52]. This will allow to determine the kaon freeze-out temperature by comparing the experimental data to lattice QCD simulations, once they become available. 


\section{Conclusions and outlook}

The precision of the present lattice results allows a comparison to experimental results for the first time in our field. From the fluctuations of conserved charges it is possible to gain information about the evolution of matter created in heavy ion collision, in particular on the chemical freeze-out. Many effects need to be understood in order to have a meaningful comparison: the theoretical and experimental efforts are progressing towards an understanding of these effects and their magnitudes. Various comparisons between theory and experiment have been performed in order to extract the freeze-out parameters of heavy ion collisions from first principles, or to predict the behavior of higher order fluctuations. While a consistent picture between baryon number and electric charge seems to emerge, an unambiguous determination of the freeze-out temperature for strangeness will help to better clarify the remaining issues.

\section{Acknowledgements}

The author acknowledges useful discussions with her colleagues and collaborators Paolo Alba, Rene Bellwied, Szabolcs Borsanyi, Zoltan Fodor, Jana Günther, Sandor Katz, Volker Koch, Valentina Mantovani-Sarti, Jorge Noronha, Jacquelyn Noronha-Hostler, Paolo Parotto, Attila Pasztor, Israel Portillo, Kalman Szabo. This material is based upon work supported by the National Science Foundation under grants no. PHY-1654219 and OAC-1531814 and by the U.S. Department of Energy, Office of Science, Office of Nuclear Physics, within the framework of the Beam Energy Scan Theory (BEST) Topical Collaboration. An award of computer time was provided by the INCITE program. This research used resources of the Argonne Leadership Computing Facility, which is a DOE Office of Science User Facility supported under Contract DE-AC02-06CH11357. The author gratefully acknowledges the use of the Maxwell Cluster and the advanced support from the Center of Advanced Computing and Data Systems at the University of Houston.

\section{References}

[1] J. Cleymans, H. Oeschler and K. Redlich, Phys. Rev. C 59, 1663 (1999) doi:10.1103/PhysRevC.59.1663 [nucl-th/9809027].

[2] A. Andronic, P. Braun-Munzinger and J. Stachel, Phys. Lett. B 673, 142 (2009) Erratum: [Phys. Lett. B 678, 516 (2009)] doi:10.1016/j.physletb.2009.02.014, 10.1016/j.physletb.2009.06.021 [arXiv:0812.1186 [nucl-th]].

[3] J. Manninen and F. Becattini, Phys. Rev. C 78, 054901 (2008) doi:10.1103/PhysRevC.78.054901 [arXiv:0806.4100 [nucl-th]].

[4] B. I. Abelev et al. [STAR Collaboration], Phys. Rev. C 81, 024911 (2010) doi:10.1103/PhysRevC.81.024911 [arXiv:0909.4131 [nucl-ex]].

[5] M. M. Aggarwal et al. [STAR Collaboration], Phys. Rev. C 83, 034910 (2011) doi:10.1103/PhysRevC.83.034910 [arXiv:1008.3133 [nucl-ex]].

[6] J. Noronha-Hostler, J. Noronha, and C. Greiner, Phys. Rev. Lett. 103, 172302 (2009), arXiv:0811.1571 [nucl-th].

[7] A. Majumder and B. Muller, Phys.Rev.Lett. 105, 252002401 (2010), arXiv:1008.1747 [hep-ph].

[8] A. Bazavov et al., Phys. Rev. Lett. 113 (2014) 072001.

[9] P. Alba et al., Phys. Rev. D96 (2017) 034517.

[10] S. Borsanyi et al., JHEP 1209 (2012) 010 doi:10.1007/JHEP09(2012)010 [arXiv:1203.4469 [hep-lat]]. 
[11] A. Andronic, P. Braun-Munzinger, K. Redlich and J. Stachel, J. Phys. Conf. Ser. 779, no. 1, 012012 (2017) doi:10.1088/1742-6596/779/1/012012 [arXiv:1611.01347 [nucl-th]].

[12] S. Borsanyi et al. [Wuppertal-Budapest Collaboration], JHEP 1009, 073 (2010) doi:10.1007/JHEP09(2010)073 [arXiv:1005.3508 [hep-lat]].

[13] A. Bazavov et al. [HotQCD Collaboration], Phys. Rev. D 90, 094503 (2014) doi:10.1103/PhysRevD.90.094503 [arXiv:1407.6387 [hep-lat]].

[14] O. Kaczmarek et al., Phys. Rev. D 83, 014504 (2011) doi:10.1103/PhysRevD.83.014504 [arXiv:1011.3130 [hep-lat]].

[15] R. Bellwied, S. Borsanyi, Z. Fodor, J. Guenther, S. D. Katz, C. Ratti and K. K. Szabo, Phys. Lett. B 751, 559 (2015) doi:10.1016/j.physletb.2015.11.011 [arXiv:1507.07510 [hep-lat]].

[16] R. Bellwied et al., Phys.Rev.Lett. 111 (2013) 202302.

[17] M. Floris, Nucl Phys. A931 (2014) 103.

[18] M. Ferraris, M. M. Giannini, M. Pizzo, E. Santopinto, and L. Tiator, Phys. Lett. B364, 231 (1995).

[19] R. Bijker, J. Ferretti, G. Galata, H. Garcia-Tecocoatzi, and E. Santopinto, Phys. Rev. D94, 074040 (2016), arXiv:1506.07469 [hep-ph].

[20] S. Capstick and N. Isgur, Phys.Rev. D34, 2809 (1986).

[21] D. Ebert, R. Faustov, and V. Galkin, Phys.Rev. D79, 114029 (2009), arXiv:0903.5183 [hep-ph].

[22] S. Borsanyi, Z. Fodor, S. D. Katz, S. Krieg, C. Ratti and K. Szabo, JHEP 1201, 138 (2012) doi:10.1007/JHEP01(2012)138 [arXiv:1112.4416 [hep-lat]].

[23] A. Bazavov et al. [HotQCD Collaboration], Phys. Rev. D 86, 034509 (2012) doi:10.1103/PhysRevD.86.034509 [arXiv:1203.0784 [hep-lat]].

[24] R. V. Gavai and S. Gupta, Phys. Rev. D 73, 014004 (2006) doi:10.1103/PhysRevD.73.014004 [hep-lat/0510044].

[25] R. Bellwied, S. Borsanyi, Z. Fodor, S. D. Katz, A. Pasztor, C. Ratti and K. K. Szabo, Phys. Rev. D 92, no. 11, 114505 (2015) doi:10.1103/PhysRevD.92.114505 [arXiv:1507.04627 [hep-lat]].

[26] H.-T. Ding, S. Mukherjee, H. Ohno, P. Petreczky and H.-P. Schadler, Phys. Rev. D 92, no. 7, 074043 (2015) doi:10.1103/PhysRevD.92.074043 [arXiv:1507.06637 [hep-lat]].

[27] V. Skokov, B. Friman and K. Redlich, Phys. Rev. C 88 (2013) 034911

[28] P. Braun-Munzinger, A. Rustamov and J. Stachel, Nucl. Phys. A 960, 114 (2017) doi:10.1016/j.nuclphysa.2017.01.011 [arXiv:1612.00702 [nucl-th]].

[29] V. Begun and M. Mackowiak-Pawlowska, arXiv:1705.01110 [nucl-th].

[30] A. Bzdak and V. Koch, Phys. Rev. C 86 (2012) 044904

[31] S. Jeon and V. Koch, Phys. Rev. Lett. 85 (2000) 2076

[32] F. Becattini, J. Steinheimer, R. Stock and M. Bleicher, Phys. Lett. B 764, 241 (2017) doi:10.1016/j.physletb.2016.11.033 [arXiv:1605.09694 [nucl-th]].

[33] J. Steinheimer, J. Aichelin and M. Bleicher, Phys. Rev. Lett. 110, no. 4, 042501 (2013) doi:10.1103/PhysRevLett.110.042501 [arXiv:1203.5302 [nucl-th]].

[34] F. Becattini, M. Bleicher, T. Kollegger, T. Schuster, J. Steinheimer and R. Stock, Phys. Rev. Lett. 111, 082302 (2013) doi:10.1103/PhysRevLett.111.082302 [arXiv:1212.2431 [nucl-th]].

[35] J. Steinheimer, V. Vovchenko, J. Aichelin, M. Bleicher and H. Stoecker, arXiv:1608.03737 [nuclth].

[36] M. Kitazawa and M. Asakawa, Phys. Rev. C 85 (2012) 021901

[37] M. Kitazawa and M. Asakawa, Phys. Rev. C 86, 024904 (2012) Erratum: [Phys. Rev. C 86, 069902 (2012)] doi:10.1103/PhysRevC.86.024904, 10.1103/PhysRevC.86.069902 
[arXiv:1205.3292 [nucl-th]].

[38] M. Nahrgang, M. Bluhm, P. Alba, R. Bellwied and C. Ratti, Eur. Phys. J. C 75 (2015) 12, 573

[39] S. Borsanyi, Z. Fodor, S. D. Katz, S. Krieg, C. Ratti and K. K. Szabo, Phys. Rev. Lett. 111 (2013) 062005

[40] S. Borsanyi, Z. Fodor, S. D. Katz, S. Krieg, C. Ratti and K. K. Szabo, Phys. Rev. Lett. 113 (2014) 052301

[41] L. Adamczyk et al. [STAR Collaboration], Phys. Rev. Lett. 112, 032302 (2014) doi:10.1103/PhysRevLett.112.032302 [arXiv:1309.5681 [nucl-ex]].

[42] L. Adamczyk et al. [STAR Collaboration], Phys. Rev. Lett. 113, 092301 (2014) doi:10.1103/PhysRevLett.113.092301 [arXiv:1402.1558 [nucl-ex]].

[43] P. Alba, W. Alberico, R. Bellwied, M. Bluhm, V. Mantovani Sarti, M. Nahrgang and C. Ratti, Phys. Lett. B 738, 305 (2014) doi:10.1016/j.physletb.2014.09.052 [arXiv:1403.4903 [hep-ph]].

[44] A. Bazavov et al., Phys. Rev. D 93, no. 1, 014512 (2016) doi:10.1103/PhysRevD.93.014512 [arXiv:1509.05786 [hep-lat]].

[45] C. Ratti, Nucl. Phys. A 956, 51 (2016) doi:10.1016/j.nuclphysa.2016.02.022 [arXiv:1601.02367 [hep-lat]].

[46] J. Gunther, R. Bellwied, S. Borsanyi, Z. Fodor, S. D. Katz, A. Pasztor and C. Ratti, EPJ Web Conf. 137, 07008 (2017) doi:10.1051/epjconf/201713707008 [arXiv:1607.02493 [hep-lat]].

[47] A. Rustamov [ALICE Collaboration], Nucl. Phys. A 967, 453 (2017) doi:10.1016/j.nuclphysa.2017.05.111 [arXiv:1704.05329 [nucl-ex]].

[48] P. Braun-Munzinger, A. Kalweit, K. Redlich and J. Stachel, Phys. Lett. B 747, 292 (2015) doi:10.1016/j.physletb.2015.05.077 [arXiv:1412.8614 [hep-ph]].

[49] A. Bazavov et al. [HotQCD Collaboration], arXiv:1708.04897 [hep-lat].

[50] L. Adamczyk et al. [STAR Collaboration], arXiv:1709.00773 [nucl-ex].

[51] P. Alba, R. Bellwied, M. Bluhm, V. Mantovani Sarti, M. Nahrgang and C. Ratti, Phys. Rev. C 92, no. 6, 064910 (2015) doi:10.1103/PhysRevC.92.064910 [arXiv:1504.03262 [hep-ph]].

[52] J. Noronha-Hostler, R. Bellwied, J. Gunther, P. Parotto, A. Pasztor, I. P. Vazquez and C. Ratti, arXiv:1607.02527 [hep-ph]. 